\title{
BOUNDED AUTOMORPHISM GROUPS OF COMPACT COMPLEX SURFACES
}

\author{
YU. G. PROKHOROV, C. A. SHRAMOV
}

\begin{abstract}
We classify compact complex surfaces whose groups of bimeromorphic selfmaps have bounded finite subgroups. We also prove that the stabilizer of a point in the automorphism group of a compact complex surface of zero Kodaira dimension, as well as the stabilizer of a point in the automorphism group of an arbitrary compact Kähler manifold of non-negative Kodaira dimension, always has bounded finite subgroups.
\end{abstract}

\section{Contents}

1. Introduction

2. Preliminaries

3. Elliptic surfaces

4. Proof of the main result 9

5. Stabilizers of points 10

6. Kähler manifolds 12

7. Abelian subgroups 13

References 14

\section{INTRODUCTION}

One says that a group $\Gamma$ has bounded finite subgroups if there exists a constant $B=B(\Gamma)$ such that every finite subgroup of $\Gamma$ has order at most $B$. Otherwise one says that $\Gamma$ has unbounded finite subgroups. In many interesting cases automorphism groups or groups of birational selfmaps of algebraic varieties have bounded finite subgroups. For instance, this is the case for non-uniruled varieties with vanishing irregularity over fields of characteristic zero (see [15, Theorem 1.8(i)]); for varieties over number fields (see [15, Theorem 1.4] and [3, Theorem 1.1]); for non-trivial Severi-Brauer surfaces over fields of characteristic zero that contain all roots of unity (see [22, Corollary 1.5]).

This work was performed at the Steklov International Mathematical Center and supported by the Ministry of Science and Higher Education of the Russian Federation (agreement no. 075-15-2019-1614). 
The main purpose of this paper is to prove the following assertion that classifies compact complex surfaces whose groups of bimeromorphic selfmaps have unbounded finite subgroups.

Theorem 1.1 (cf. [17, Lemma 3.5]). Let $S$ be a compact complex surface of non-negative Kodaira dimension. Suppose that the group $\operatorname{Bim}(S)$ of bimeromorphic selfmaps of $S$ has unbounded finite subgroups. Then $S$ is bimeromorphic to a surface of one of the following types:

- a complex torus;

- a bielliptic surface;

- a Kodaira surface;

- a surface of Kodaira dimension 1.

Moreover, in the first three cases the group $\operatorname{Bim}(S)$ always has unbounded finite subgroups. In the fourth case the group $\operatorname{Bim}(S)$ has bounded finite subgroups if and only if this holds for its subgroup that consists of all selfmaps preserving the fibers of the pluricanonical fibration of $S$.

One of the main steps in the proof of Theorem 1.1 is the following assertion which, as we think, is interesting on its own.

Proposition 1.2. Let $S$ be a minimal compact complex surface of Kodaira dimension 1. Consider the pluricanonical fibration $\phi: S \rightarrow C$. Then the image of the group $\operatorname{Aut}(S)$ in $\operatorname{Aut}(C)$ is finite.

Also, in this paper we prove the following result. For a complex manifold $X$ and a point $P \in X$, by $\operatorname{Aut}(X ; P)$ we denote the stabilizer of $P$ in the group $\operatorname{Aut}(X)$.

Proposition 1.3. There exists a constant $B$ such that for every compact complex surface $S$ of Kodaira dimension 0 , every point $P \in S$, and every finite subgroup $G \subset \operatorname{Aut}(S ; P)$ the order of the group $G$ is at most $B$.

Remark 1.4. Let $S$ be a compact complex surface of Kodaira dimension 1, and let $P$ be a point on $S$. We do not know whether the group $\operatorname{Aut}(S ; P)$ always has bounded finite subgroups. It is easy to show that this holds at least when the fiber of the pluricanonical fibration of $S$ passing through $P$ is non-singular and reduced at $P$.

Under an additional assumption that a manifold is Kähler we can prove boundedness of finite subgroups for stabilizers of points in the automorphism groups in arbitrary dimension.

Theorem 1.5. Let $X$ be a compact Kähler manifold of nonnegative Kodaira dimension, and let $P$ be a point on $X$. Then the group $\operatorname{Aut}(X ; P)$ has bounded finite subgroups. 
We will prove one more assertion whose structure is similar to that of Proposition 1.3, it completes some of the results of the paper [16].

Proposition 1.6 (cf. [16, Corollary 8.10]). There exists a constant J such that for every compact complex surface $S$ of Kodaira dimension 0 and every finite subgroup $G \subset \operatorname{Bim}(S)$ there is an abelian subgroup of index at most $J$ in $G$.

In Section 2 we prove several auxiliary assertions. In Section 3 we study automorphism groups of elliptic fibrations and prove Proposition 1.2. In Section 4 we prove Theorem 1.1. In Section 5 we prove Proposition 1.3. In Section 6 we prove Theorem 1.5. In Section 7 we prove Proposition 1.6.

We use the following notation and conventions. A complex manifold is a smooth irreducible complex space. A morphism is a holomorphic map of complex spaces; a fibration is a morphism with connected fibers of positive dimension. A typical fiber of a fibration $\phi: X \rightarrow Y$ is a fiber over a point of some non-empty subset of the form $Y \backslash \Sigma$, where $\Sigma$ is a closed (analytic) subset in $Y$. By $\mathscr{K}_{X}$ we denote the canonical line bundle on a (compact) complex manifold $X$, and by $\varkappa(X)$ we denote the Kodaira dimension of $X$. By $T_{P}(X)$ we denote the tangent space to a complex manifold $X$ at a point $P \in X$. Given a complex manifold $X$ and a morphism $\phi: X \rightarrow Y$ which is equivariant with respect to the group $\operatorname{Aut}(X)$, we denote by $\operatorname{Aut}(X)_{\phi}$ the group that consists of all automorphisms mapping every fiber of $\phi$ to itself.

We are grateful to F. Campana, S. Nemirovski, and W. Sawin for useful discussions. Special thanks go to the referee for his remarks.

\section{Preliminaries}

In this section we prove some auxiliary results on compact complex surfaces. The following assertion is well-known.

Lemma 2.1. Let $S$ be a compact complex surface. Suppose that there are two divisors $C_{1}$ and $C_{2}$ on $S$ such that $C_{1}^{2} \geqslant 0$ and $C_{1} \cdot C_{2}>0$. Then the surface $S$ is projective.

Proof. For $n \gg 0$ one has

$$
\left(n C_{1}+C_{2}\right)^{2}=n^{2} C_{1}^{2}+2 n C_{1} \cdot C_{2}+C_{2}^{2}>0,
$$

so that $S$ is projective by [4, Theorem IV.6.2].

Recall that a compact complex surface $S$ is called minimal if it does not contain smooth rational curves with self-intersection -1 . For every compact complex surface $S$ there exists a minimal surface $S^{\prime}$ bimeromorphic to $S$, see for instance [4, Theorem III.4.5]. 
Furthermore, if $\varkappa(S) \geqslant 0$, then the minimal surface $S^{\prime}$ is unique, see [4, Proposition III.4.6]; in this case there is a bimeromorphic morphism $\pi: S \rightarrow S^{\prime}$. Recall also that there exists a Kodaira-Enriques classification of minimal compact complex surfaces, see [4, Chapter VI].

Lemma 2.2 (see for instance [16, Proposition 3.5]). Let $S$ be a nonruled minimal compact complex surface. Then $\operatorname{Bim}(S)=\operatorname{Aut}(S)$.

Lemma 2.3. Let $S$ be a complex manifold, and let $\phi: S \rightarrow C$ be a morphism that is equivariant with respect to the group $\operatorname{Aut}(S)$. Let

$$
F=\phi^{*}(c), \quad c \in C,
$$

be a fiber of the morphism $\phi$. Suppose that there is an irreducible component $F_{1} \subset \operatorname{Supp}(F)$ of multiplicity 1 in $F$. Then every finite subgroup of $\operatorname{Aut}(S)_{\phi}$ acts faithfully on $F$.

Proof. Suppose that the induced action on $F$ of some non-trivial finite subgroup $G \subset \operatorname{Aut}(S)_{\phi}$ is trivial; in particular, $G$ preserves the irreducible component $F_{1}$ and acts trivially on it. Choose a point $P \in F_{1}$ such that the morphism $\phi$ is smooth at $P$. The group $G$ acts non-trivially in the tangent space $T_{P}(S)$, see for instance [1, §2.2] or [16, Corollary 4.2]. On the other hand, $G$ acts trivially on the subspace $T_{P}\left(F_{1}\right) \subset T_{P}(S)$. Since the morphism $\phi$ is smooth at $P$, the differential

$$
d \phi: T_{P}(S) \longrightarrow T_{\phi(P)}(C)
$$

is a surjective linear map whose kernel is identified with $T_{P}\left(F_{1}\right)$. Moreover, this map is $G$-equivariant, where the action of $G$ on $C$ is taken to be trivial. Since $G$ acts trivially on the tangent space $T_{\phi(P)}(C)$, we conclude that the action of $G$ on the tangent space $T_{P}(S)$ is trivial as well. The obtained contradiction shows that the action of $G$ on $F$ cannot be trivial.

Recall that for a primary Kodaira surface the algebraic reduction gives a morphism onto an elliptic curve, and all of its fibers are elliptic curves. This morphism is equivariant with respect to the automorphism group of the surface. For a secondary Kodaira surface the algebraic reduction is a morphism to a rational curve whose typical fiber is an elliptic curve.

Lemma 2.4. Let $S$ be a primary (respectively, secondary) Kodaira surface, and let $\phi: S \rightarrow C$ be its algebraic reduction. Then the image $\Gamma$ of the group $\operatorname{Aut}(S)$ in $\operatorname{Aut}(C)$ has order at most 6 (respectively, 24).

Proof. If $S$ is a primary Kodaira surface, then the group $\Gamma$ does not contain elements that act on the elliptic curve $C$ by translations, 
see [21, Corollary 3.3]. Since the automorphism group of an elliptic curve is a semi-direct product of the subgroup of translations with a cyclic group of order at most 6 , we conclude that the order of $\Gamma$ is at most 6 .

Let $S$ be a secondary Kodaira surface, and let $\phi: S \rightarrow \mathbb{P}^{1}$ be its algebraic reduction. Then $\phi$ has either 3 or 4 multiple fibers, see for instance the proof of [16, Lemma 7.1]. Thus $\Gamma$ is isomorphic to a subgroup of the symmetric group on four elements. In particular, the order of $\Gamma$ is at most 24.

Lemma 2.5. Let $S$ be a primary Kodaira surface, and let $\phi: S \rightarrow C$ be its algebraic reduction. Let $G \subset \operatorname{Aut}(S)_{\phi}$ be a non-trivial subgroup of finite order. Then $G$ acts on $S$ without fixed points.

Proof. Without loss of generality, we may assume that the group $G$ is cyclic. Let $\gamma$ be its generator. Suppose that $G$ has a fixed point $P \in S$. Let $F$ be the fiber of $\phi$ passing through $P$. Recall that the fiber $F$ is irreducible and non-multiple, see [4, § V.5]. By Lemma 2.3 the automorphism $\gamma$ acts non-trivially on the elliptic curve $F$. Suppose that $\gamma$ fixes some point $P$ on $F$. Note that the morphism $\phi$ is smooth at $P$ (as well as at any other point on $S$ ). Arguing as in the proof of Lemma 2.3, we see that the differential

$$
d \phi: T_{P}(S) \longrightarrow T_{\phi(P)}(C)
$$

allows us to identify the two-dimensional representation $T_{P}(S)$ of the cyclic group $G$ with the direct sum $T_{P}(F) \oplus T$, where the action of $G$ on the subspace $T \cong T_{\phi(P)}(C)$ is trivial. By [16, Corollary 4.7] there exists a (compact) curve $D \subset S$ that consists of fixed points of $\gamma$, such that $D$ passes through $P$ and the tangent space to $D$ at $P$ coincides with the subspace $T \subset T_{P}(S)$. Therefore, we have $\phi(D)=C$. Since the surface $S$ is not projective, this gives a contradiction with Lemma 2.1.

We will use the following classical theorem proved by H. Minkowski.

Theorem 2.6 (see for instance [20, Theorem 1]). For every positive integer $n$ the group $\mathrm{GL}_{n}(\mathbb{Q})$ has bounded finite subgroups.

Corollary 2.7. For every positive integer $n$ there exists a constant $B_{T}(n)$ such that for every $n$-dimensional complex torus $S$, every point $P$ on $S$, and every finite subgroup $G \subset \operatorname{Aut}(S ; P)$, the order of $G$ is at most $B_{T}(n)$.

Proof. It is well-known that $\operatorname{Aut}(S ; P)$ is isomorphic to a subgroup of $\mathrm{GL}_{2 n}(\mathbb{Z})$; see for instance [16, Theorem 8.4]. Therefore, the assertion follows from Theorem 2.6 . 
Corollary 2.8. There exists a constant $B_{K 3}$ such that for every compact complex surface $S$ which is either a $K 3$ surface or an Enriques surface, and for every finite subgroup $G \subset \operatorname{Aut}(S)$, the order of $G$ is at most $B_{K 3}$.

Proof. Let $S$ be either a $K 3$ surface or an Enriques surface. Consider the representation of $\operatorname{Aut}(S)$ in the cohomology group

$$
\rho: \operatorname{Aut}(S) \rightarrow \mathrm{GL}\left(H^{*}(S, \mathbb{Q})\right) .
$$

In both cases we have to consider the dimension of the vector space $H^{*}(S, \mathbb{Q})$ does not exceed 24 , see for instance [4, Table 10]. By Theorem [2.6] the orders of finite subgroups in the image of the representation $\rho$ are bounded by some constant that does not depend on $S$. On the other hand, if $S$ is a $K 3$ surface, then the representation $\rho$ is faithful (see for instance [7, Proposition 15.2.1]). If $S$ is an Enriques surface, then the kernel of $\rho$ contains at most 4 elements (see [13]).

Finite groups acting on $K 3$ surfaces are well studied, see [14] and references therein. This classification can be used to obtain an adequate bound for the constant $B_{K 3}$ from Corollary 2.8.

The following theorem is well-known.

Theorem 2.9 (see for instance [23, Corollary 14.3]). Let $S$ be a compact complex surface of Kodaira dimension 2. Then the group $\operatorname{Aut}(S)$ is finite.

\section{ElLiptic SURFACES}

In this section we prove Proposition 1.2 .

To describe fibers of elliptic fibrations we will use the standard notation for types of degenerate fibers in the Kodaira classification, see [4, §V.7] or [12, § I.4]. Recall that a fibration $\phi: S \rightarrow C$ with compact fibers, where $S$ is a smooth (but possibly non-compact) complex surface and $C$ is a smooth (but possibly non-compact) curve, is called relatively minimal if the fibers of $\phi$ do not contain smooth rational curves with self-intersetion -1 .

Given an elliptic fibration $\phi: S \rightarrow C$, one can consider the function $J$ that associates to a point $c \in C$ the value of the $j$-invariant of the fiber $F=\phi^{*} c$, provided that the fiber $F$ is smooth. It is straightforward to see that $J$ is a meromorphic function on $C$.

Lemma 3.1. Let $\Delta \subset \mathbb{C}$ be the unit disc. Let $S$ be a smooth (noncompact) complex surface, and let $\phi: S \rightarrow \Delta$ be a fibration with compact fibers whose typical fiber is an elliptic curve. Suppose that $\phi$ is relatively minimal. Consider the fiber $F=\phi^{*} \mathbf{0}$ over the point $\mathbf{0} \in \Delta$. 
Suppose that the fiber $F$ is non-multiple, and has type $I_{b}$ or $I_{b}^{*}$ for some $b \geqslant 1$. Then the function $J$ has a pole at the point $\mathbf{0 .}$

Proof. Since the fiber $F$ is non-multiple, at least one of its irreducible components is reduced (see [4, §V.7]). Hence in a small neighborhood of the point $\mathbf{0}$ the fibration $\phi$ admits an analytic section. Thus we can assume that $\phi$ is taken to the Weierstrass form, see [12, Lecture II]. Now the assertion follows from [12, Table IV.3.1] (cf. [4, §V.10, Table 6] and [12, Proposition VI.1.1]).

Recall that a fibration is called isotrivial if its fibers over the points of some dense open subset are isomorphic to each other.

Lemma 3.2 (cf. [19, §2.1]). Let $\Delta \subset \mathbb{C}$ be the unit disc. Let $S$ be a smooth (non-compact) complex surface, and let $\phi: S \rightarrow \Delta$ be a fibration with compact fibers whose typical fiber is an elliptic curve. Suppose that $\phi$ is relatively minimal and isotrivial. Consider the fiber $F=\phi^{*} \mathbf{0}$ over the point $\mathbf{0} \in \Delta$. If $F$ is a non-multiple fiber, then it cannot be of type $I_{b}$ or $I_{b}^{*}$ for $b \geqslant 1$; if $F$ is a multiple fiber, then it can only have type ${ }_{m} I_{0}$ for some $m \geqslant 2$.

Proof. Since the fibration $\phi$ is isotrivial, the $j$-invariant of its fibers is a constant function on the set $\Delta \backslash \Lambda$, where $\Lambda$ is the set of images of all multiple fibers of $\phi$. In particular, this function cannot have a pole at a point of $\Delta \backslash \Lambda$. Therefore, the assertion about non-multiple fibers follows from Lemma 3.1 .

Now suppose that the fiber $F$ is multiple. According to [4, § V.7], it can only have type ${ }_{m} I_{r}$, where $r \geqslant 0$ and $m \geqslant 2$. We need to exclude the case $r \geqslant 1$. To do this, apply the standard construction from [4, §III.10] or [9, pp. 571-572]: it gives an isotrivial relatively minimal elliptic fibration over $\Delta$ with a non-multiple fiber of type $I_{r^{\prime}}$ for some $r^{\prime}>0$. The latter gives a contradiction with what we already proved above.

We will need a detailed information on automorphism groups of compact complex surafces of Kodaira dimension 1 (similar computations were used in the proofs of [16, Lemma 8.2] and [17, Lemma 3.3]).

Lemma 3.3. Let $S$ be a minimal compact complex surface of Kodaira dimension 1. Consider the pluricanonical fibration $\phi: S \rightarrow C$ and suppose that $C \cong \mathbb{P}^{1}$. Then the image $\Gamma$ of the group $\operatorname{Aut}(S)$ in $\operatorname{Aut}(C)$ is finite.

Proof. The group $\Gamma$ acts faithfully on $\mathbb{P}^{1}$. Assume that $\Gamma$ is infinite. Then there exists a point $P \in \mathbb{P}^{1}$ with an infinite $\Gamma$-orbit $\Xi_{P} \subset \mathbb{P}^{1}$ (indeed, if the orbit of every point is finite, $\Gamma$ contains a subgroup of 
finite index that fixes three points on $\mathbb{P}^{1}$ ). The fibers of $\phi$ over all points of $\Xi_{P}$ are isomorphic to each other. Since the $j$-invariant of the fibers of $\phi$ is a meromorphic function on $\mathbb{P}^{1}$, we conclude that this function is constant, so that the elliptic fibration $\phi$ is isotrivial. In particular, by Lemma 3.2 all multiple fibers of $\phi$ have type ${ }_{m} I_{0}$.

Suppose that $\phi$ has at least one singular non-multiple fiber. Since the group $\Gamma$ is infinite, $\phi$ has at most two singular non-multiple fibers; indeed, otherwise $\Gamma$ would have a finite invariant subset in $\mathbb{P}^{1}$ of cardinality at least 3 , which is impossible for a subgroup of $\operatorname{Aut}\left(\mathbb{P}^{1}\right)$. By Lemma 3.2 and Kodaira classification the topological Euler characteristic of any singular non-multiple fiber of $\phi$ is positive and does not exceed 10. Thus, one has

$$
0<\chi_{\text {top }}(S) \leqslant 20 .
$$

By Noether's formula we obtain

$$
\chi\left(\mathscr{O}_{S}\right)=\frac{1}{12}\left(\mathrm{c}_{1}(S)^{2}+\chi_{\mathrm{top}}(S)\right)=\frac{\chi_{\mathrm{top}}(S)}{12},
$$

which implies that $\chi\left(\mathscr{O}_{S}\right)=1$.

Let $F_{i}$ be all multiple fibers of $\phi$ (considered with reduced structure), and let $m_{i}$ be the multiplicity of $F_{i}$. By the canonical bundle formula (see [4, Theorem V.12.1]) one has

$$
\mathscr{K}_{S} \sim \phi^{*}\left(\mathscr{K}_{\mathbb{P} 1} \otimes \mathcal{L}\right) \otimes \mathscr{O}_{S}\left(\sum\left(m_{i}-1\right) F_{i}\right),
$$

where $\mathcal{L}$ is some line bundle of degree $\chi\left(\mathscr{O}_{S}\right)=1$ on $\mathbb{P}^{1}$. Keeping in mind that $\varkappa(S)=1$, we obtain

$$
-1+\sum\left(1-1 / m_{i}\right)=\operatorname{deg}\left(\mathscr{K}_{\mathbb{P}^{1}} \otimes \mathcal{L}\right)+\sum\left(1-1 / m_{i}\right)>0 .
$$

Hence $\phi$ has at least two multiple fibers. Together with a non-multiple singular fiber that exists by assumption, this gives a finite $\Gamma$-invariant subset of $\mathbb{P}^{1}$ containing at least three points. The latter means that the group $\Gamma$ is finite, which contradicts our assumption.

Therefore, $\phi$ has no non-multiple singular fibers. Hence $\chi_{\text {top }}(S)=0$, so that Noether's formula gives $\chi\left(\mathscr{O}_{S}\right)=0$. By the canonical bundle formula one has

$$
-2+\sum\left(1-1 / m_{i}\right)>0
$$

where $m_{i}$ are the multiplicities of the multiple fibers of $\phi$. This implies that $\phi$ has at least three multiple fibers. Thus we again obtain a finite $\Gamma$-invariant subset in $\mathbb{P}^{1}$ containing at least three points, which gives a contradiction.

Now we are ready to consider the general case. 
Proof of Proposition 1.2. Let $\Gamma$ be the image of the group $\operatorname{Aut}(S)$ in $\operatorname{Aut}(C)$. The group $\Gamma$ acts faithfully on the curve $C$. In particular, if the genus $g(C)$ is at least 2 , then $\Gamma$ is finite, because the whole group $\operatorname{Aut}(C)$ is finite. On the other hand, by Lemma 3.3 we can assume that $g(C) \neq 0$. Therefore, it remains to consider the case $g(C)=1$.

If $\phi$ is not a smooth morphism, then the group $\Gamma$ has a non-empty finite invariant subset in $C$. Since $g(C)=1$, this implies that $\Gamma$ is finite.

Therefore, we can assume that the morphism $\phi$ is smooth, and thus all of its fibers are elliptic curves. In this case we have $\chi_{\text {top }}(S)=0$. By Noether's formula one has $\chi\left(\mathscr{O}_{S}\right)=0$. By the canonical bundle formula we obtain

$$
\mathscr{K}_{S} \sim \phi^{*}\left(\mathscr{K}_{C} \otimes \mathcal{L}\right),
$$

where $\mathcal{L}$ is some line bundle of degree $\chi\left(\mathscr{O}_{S}\right)=0$ on $C$. Hence the Kodaira dimension of $S$ is non-positive, which contradicts our assumption.

\section{Proof of the MAIN Result}

In this section we prove Theorem 1.1.

Proof of Theorem 1.1. We may assume that the surface $S$ is minimal. In this case $\operatorname{Bim}(S)=\operatorname{Aut}(S)$ by Lemma 2.2 .

Suppose that $\varkappa(S)=0$. Let us use the classification of minimal compact complex surfaces of Kodaira dimension 0, see [4, Chapter VI]: the surface $S$ is either a $K 3$ surface, or an Enriques surface, or a complex torus, or a bielliptic surface, or a Kodaira surface. If $S$ is either a $K 3$ surface or an Enriques surface, then the group $\operatorname{Aut}(S)$ has bounded finite subgroups, see Corollary 2.8 or [16, Lemma 8.8]. On the other hand, if $S$ is either a complex torus, or a bielliptic surface, or a Kodaira surface, then the group $\operatorname{Aut}(S)$ has unbounded finite subgroups, see [21, Theorem 1.1(i)].

Suppose that $\varkappa(S)=1$. Consider the pluricanonical map $\phi: S \rightarrow C$. Since $\phi$ is equivariant with respect to the $\operatorname{group} \operatorname{Aut}(S)$, there is an exact sequence of groups

$$
1 \rightarrow \operatorname{Aut}(S)_{\phi} \rightarrow \operatorname{Aut}(S) \rightarrow \Gamma,
$$

where $\Gamma$ is a subgroup of $\operatorname{Aut}(C)$. According to Corollary 1.2, the group $\Gamma$ is finite. This implies that the group $\operatorname{Aut}(S)$ has bounded finite subgroups if and only if this holds for the group $\operatorname{Aut}(S)_{\phi}$.

Finally, if $\varkappa(S)=2$, then the assertion follows from Theorem 2.9. 
In the case of compact Kähler surfaces one can make Theorem 1.1 a bit more precise. Recall that all Kodaira surfaces are non-Kähler. This follows from the observation that the first Betti number of a (primary or secondary) Kodaira surface is always odd, see [4, §V.5.B.Ib]. On the other hand, the first Betti number of a compact Kähler surface must be even, see [4, Theorem IV.3.1]. Thus, Theorem 1.1 implies

Corollary 4.1. Let $S$ be a compact Kähler surface with $\varkappa(S) \geqslant 0$. Suppose that the group $\operatorname{Bim}(S)$ has unbounded finite subgroups. Then $S$ is bimeromorphic to a surface of one of the following types:

- a complex torus;

- a bielliptic surface;

- a surface of Kodaira dimension 1.

Moreover, in the first two cases the group $\operatorname{Bim}(S)$ always has unbounded finite subgroups.

Remark 4.2. While Theorem 1.1 gives a classification of compact complex surfaces of non-negative Kodaira dimension whose bimeromorphic automorphism groups have bounded finite subgroups, a similar question can be asked about automorphism groups of minimal surfaces of negative Kodaira dimension. In this case it makes sense to investigate finiteness of the whole automorphism group. The automorphism groups of ruled surfaces were studied in [11]; in particular, necessary and sufficient conditions for their finiteness are known. In higher dimensions, there are some results on finiteness of automorphism groups of smooth Fano threefolds (see [5]) and certain rational affine varieties (see e.g. [18]).

\section{StABILIZERS OF POINTS}

In this section we prove Proposition 1.3. We start by deriving two corollaries from the results of Section 2 .

Corollary 5.1. Let $S$ be a bielliptic surface, and let $P$ be a point on $S$. Then the stabilizer $\operatorname{Aut}(S ; P)$ has order at most 36.

Proof. Consider the Albanese map $\phi: S \rightarrow C$. This map is equivariant with respect to the group $\operatorname{Aut}(S)$. Recall that $\chi_{\text {top }}(S)=0$, so that by Noether's formula we have $\chi\left(\mathscr{O}_{S}\right)=0$. Therefore, it follows from the canonical bundle formula that $\phi$ has no multiple fibers.

Let $F$ be the fiber of the morphism $\phi$ passing through $P$. Consider the natural homomorphism

$$
\sigma: \operatorname{Aut}(S ; P) \rightarrow \operatorname{Aut}(C) \times \operatorname{Aut}(F) .
$$


It follows from Lemma 2.3 that the homomorphism $\sigma$ is injective. Furthermore, its image is contained in the subgroup

$$
\operatorname{Aut}(C ; \phi(P)) \times \operatorname{Aut}(F ; P) \subset \operatorname{Aut}(C) \times \operatorname{Aut}(F) .
$$

Since both $C$ and $F$ are elliptic curves, we see that

$$
|\operatorname{Aut}(S ; P)| \leqslant|\operatorname{Aut}(C ; P) \times \operatorname{Aut}(F ; P)| \leqslant 36 \text {. }
$$

Corollary 5.2. Let $S$ be a Kodaira surface, and let $P$ be a point on $S$. Let $G$ be a finite subgroup in the stabilizer $\operatorname{Aut}(S ; P)$. Then $G$ has order at most 6 if $S$ is a primary Kodaira surface, and at most 36 if $S$ is a secondary Kodaira surface.

Proof. First suppose that $S$ is a primary Kodaira surface. Consider the algebraic reduction $\phi: S \rightarrow C$, and set $G^{\prime}=G \cap \operatorname{Aut}(S)_{\phi}$. By Lemma 2.4 the subgroup $G^{\prime} \subset G$ has index at most 6 . Let $F$ be a fiber of the morphism $\phi$ passing through the point $P$. By Lemma 2.5 every non-trivial element of the group $G^{\prime}$ acts on $F$ without fixed points. Therefore, the group $G^{\prime}$ is trivial, and $|G| \leqslant 6$.

Now suppose that $S$ is a secondary Kodaira surface. Then there exists a canonical finite cover

$$
\theta: \tilde{S} \rightarrow S
$$

where $\tilde{S}$ is a primary Kodaira surface, and the degree of $\theta$ is at most 6 . In fact, in this case the class $\left[\mathscr{K}_{S}\right] \in H^{2}(S, \mathbb{Z})$ of the canonical line bundle $\mathscr{K}_{S}$ is an $n$-torsion element with $n=2,3$, 4, or 6 , see [4, §VI.1]. By the universal coefficient theorem it defines an $n$ torsion element in $H_{1}(S, \mathbb{Z})$ and so there exists a canonically defined subgroup of index $n$ in the fundamental group $\pi_{1}(S)$ which in turns defines our cover $\theta$. In other words, the surface $\tilde{S}$ is the analytic spectrum $\operatorname{Spec}_{\mathrm{a}} \mathrm{n}(\mathcal{R})$ of the canonical $\mathscr{O}_{S^{-}}$algebra

$$
\mathcal{R}=\oplus_{i=0}^{n-1} \mathscr{K}_{S}^{\otimes i} .
$$

Since $\theta$ is canonically defined, there is a surjective homomorphism $\operatorname{Aut}(\tilde{S}) \rightarrow \operatorname{Aut}(S)$. In particular, there exists a finite sub$\operatorname{group} \tilde{G} \subset \operatorname{Aut}(\tilde{S})$ with a surjective homomorphism onto $G$. Let $\tilde{P}_{0}$ be one of the preimages of the point $P$ on $\tilde{S}$. Then $\tilde{G}$ contains a subgroup $\tilde{G}_{0}$ of index at most 6 such that $\tilde{G}$ fixes the point $\tilde{P}_{0}$. As we already proved above, one has $\left|\tilde{G}_{0}\right| \leqslant 6$, and hence

$$
|G| \leqslant|\tilde{G}| \leqslant 6\left|\tilde{G}_{0}\right| \leqslant 36 .
$$

Remark 5.3. We do not know if the bounds obtained in Corollaries 5.1 and 5.2 are sharp. 
Now we will complete the case of Kodaira dimension 0 .

Proof of Proposition 1.3. Let $S$ be a compact complex surface of Kodaira dimension 0 . Consider the minimal model $S^{\prime}$ of the surface $S$. Then $\operatorname{Aut}(S) \subset \operatorname{Bim}\left(S^{\prime}\right)$; hence by Lemma 2.2 there is an embedding $\operatorname{Aut}(S) \subset \operatorname{Aut}\left(S^{\prime}\right)$. Consider the bimeromorphic morphism $\pi: S \rightarrow S^{\prime}$. Since the minimal model $S^{\prime}$ is unique, the morphism $\pi$ is equivariant with respect to the group $\operatorname{Aut}(S)$. Therefore, the image $\pi(P)$ of the point $P$ is invariant under the group $\operatorname{Aut}(S ; P)$. Thus we can assume from the very beginning that the surface $S$ is minimal.

Let us use the classification of minimal compact complex surfaces of Kodaira dimension 0. If $S$ is either a $K 3$ surface or an Enriques surface, the assertion follows from Corollary 2.8. If $S$ is a complex torus, the assertion follows from Corollary 2.7. If $S$ is a bielliptic surface, the assertion follows from Corollary 5.1. Finally, if $S$ is a Kodaira surface, the assertion follows from Corollary 5.2 .

\section{KÄHLER MANIFOLDS}

In this section we prove Theorem 1.5. Given a complex variety $X$, by $\operatorname{Aut}^{0}(X)$ we will denote the connected component of identity in the complex Lie group $\operatorname{Aut}(X)$.

Theorem 6.1 (see [6, Corollary 5.11]). Let $X$ be a compact Kähler manifold of non-negative Kodaira dimension. Then the group $\operatorname{Aut}^{0}(X)$ either is trivial, or is a complex torus.

Lemma 6.2. Let $X$ be a compact complex manifold. Suppose that $X$ is non-trivially acted on by a complex torus $T$. Then $T$ has no fixed points on $X$.

Proof. The action of $T$ on $X$ is given by a morphism

$$
\Psi: T \times X \rightarrow X
$$

Suppose that some point $P \in X$ is fixed by $T$. Then the image $\Psi(T \times\{P\})$ is a point. On the other hand, since the action of $T$ on $X$ is non-trivial, for a typical point $Q \in X$ the image $\Psi(T \times\{Q\})$ is not a point. This is impossible by the rigidity theorem, see [10, Theorem 5.23].

Corollary 6.3. Let $X$ be a compact Kähler manifold of non-negative Kodaira dimension, and let $P$ be a point on $X$. Then the group

$$
\operatorname{Aut}^{0}(X ; P)=\operatorname{Aut}(X ; P) \cap \operatorname{Aut}^{0}(X)
$$

is finite. Moreover, there exists a constant $B=B(X)$ that does not depend on $P$, such that $\left|\operatorname{Aut}^{0}(X ; P)\right| \leqslant B$. 
Proof. If the group $\operatorname{Aut}^{0}(X)$ is trivial, there is nothing to prove. Thus we will assume that $\operatorname{Aut}^{0}(X)$ is non-trivial, and hence it is a complex torus by Theorem 6.1.

Suppose that the group $\operatorname{Aut}^{0}(X ; P)$ is infinite. Since it is a closed subgroup in $\operatorname{Aut}^{0}(X)$, it contains some complex torus $T$. Thus $T$ acts on $X$ with the fixed point $P$, which contradicts Lemma 6.2. Therefore, the group $\operatorname{Aut}^{0}(X ; P)$ is finite.

Now consider the incidence relation

$$
\Xi=\{(\sigma, Q) \mid \sigma(Q)=Q\} \subset \operatorname{Aut}^{0}(X) \times X,
$$

and denote by $\pi: \Xi \rightarrow X$ the projection on the second factor. Then $\Xi$ is a (possibly reducible) compact complex space, and a fiber of $\pi$ over a point $P$ is exactly the subgroup $\operatorname{Aut}^{0}(X ; P)$. The projection $\pi$ is a proper map. Since the fibers of $\pi$ are finite, we conclude that the map $\pi$ is finite. We claim that the number of points in the fibers of $\pi$ is bounded by some constant $B=B(X)$. Indeed, since the number of irreducible components of $\Xi$ is finite, we may replace $\Xi$ by its irreducible component. Now the number of points in the fiber is bounded by the degree of the map $\pi: \Xi \rightarrow \pi(\Xi)$.

Now we are ready to prove the main result of this section.

Proof of Theorem 1.5. According to Corollary 6.3, the intersection $\operatorname{Aut}(X ; P) \cap \operatorname{Aut}^{0}(X)$ has bounded order. Hence it is enough to check that the image of $\operatorname{Aut}(X ; P)$ in the quotient group

$$
\Upsilon=\operatorname{Aut}(X) / \operatorname{Aut}^{0}(X)
$$

has bounded finite subgroups. On the other hand, by [8, Lemma 3.1] the whole group $\Upsilon$ has bounded finite subgroups.

Corollary 6.4. Let $S$ be a minimal compact Kähler surface of Kodaira dimension 1. Suppose that the pluricanonical fibration $\phi: S \rightarrow C$ has a singular fiber. Then the group $\operatorname{Bim}(S)$ has bounded finite subgroups.

Proof. One has $\operatorname{Bim}(S)=\operatorname{Aut}(S)$ by Lemma 2.2. Consider the set $\Sigma \subset S$ of singular points of all singular fibers of $\phi$, and choose a point $P \in \Sigma$. Since $\phi$ is equivariant with respect to the group $\operatorname{Aut}(S)$, this group acts on $\Sigma$. Hence the stabilizer $\operatorname{Aut}(S ; P)$ of the point $P$ has index at most $|\Sigma|$ in $\operatorname{Aut}(S)$. Now it remains to apply Theorem 1.5 .

\section{Abelian subgroups}

In this section we prove Proposition 1.6. We start with one of its particular cases that we treat using the results of Section 2 . 
Corollary 7.1. Let $S$ be a Kodaira surface, and let $G$ be a finite subgroup in $\operatorname{Aut}(S)$. Then $G$ contains an abelian subgroup of index at most 6 .

Proof. First suppose that $S$ is a primary Kodaira surface. Consider the algebraic reduction $\phi: S \rightarrow C$, and set $G^{\prime}=G \cap \operatorname{Aut}(S)_{\phi}$. By Lemma 2.4 the subgroup $G^{\prime} \subset G$ has index at most 6. By Corollary 5.2 the group $G^{\prime}$ acts by translations on an elliptic curve that is a fiber of the morphism $\phi$. In particular, this group is abelian.

Now suppose that $S$ is a secondary Kodaira surface. As in the proof of Corollary 5.2, there exists a primary Kodaira surface $\tilde{S}$ and a finite subgroup $\tilde{G} \subset \operatorname{Aut}(\tilde{S})$ with a surjective homomorphism onto $G$. According to what we proved above, the group $\tilde{G}$ contains an abelian subgroup $\tilde{G}^{\prime}$ of index at most 6 . Its image in $G$ will also be an abelian subgroup of index at most 6 .

Now we prove a general assertion.

Prove of Proposition 1.6. Let $S$ be a compact complex surface of Kodaira dimension 0 . Replace $S$ by its minimal model. Then $\operatorname{Bim}(S)=\operatorname{Aut}(S)$ by Lemma 2.2.

Let us use the classification of minimal compact complex surfaces of Kodaira dimension 0. If $S$ is either a $K 3$ surface or an Enriques surface, then the assertion follows from Corollary 2.8, If $S$ is a complex torus, then the assertion follows from Corollary 2.7, since in this case the quotient of the group $\operatorname{Aut}(S)$ by the abelian subgroup acting by translations on $S$ is isomorphic to the stabilizer of (any) point of $S$. If $S$ is a Kodaira surface, then the assertion follows from Corollary 7.1 Finally, if $S$ is a bielliptic surface, then the assertion follows from the explicit description of the group $\operatorname{Aut}(S)$, see [2, Table 3.2]: according to this description $\operatorname{Aut}(S)$ contains a subgroup of index at most 24 isomorphic to the group of points of an elliptic curve.

\section{REFERENCES}

[1] Akhiezer D. N. Lie group actions in complex analysis. Aspects of Mathematics, E27. - Braunschweig: Friedr. Vieweg \& Sohn, 1995. - P. viii+201. - ISBN: 3-528-06420-X - Access mode: http://dx.doi.org/10.1007/978-3-322-80267-5.

[2] Bennett C., Miranda R. The automorphism groups of the hyperelliptic surfaces // Rocky Mountain J. Math. — 1990. — Vol. 20, no. 1. — P. 31-37. Access mode: https://doi.org/10.1216/rmjm/1181073156.

[3] Birkar C. Singularities of linear systems and boundedness of Fano varieties // arXiv:1609.05543. - 2016.

[4] Compact complex surfaces / W. P. Barth, K. Hulek, Ch. A. M. Peters, A. Van de Ven. - Second edition. - Berlin : Springer-Verlag, 2004. - Vol. 4 
of Ergebnisse der Mathematik und ihrer Grenzgebiete. 3. Folge. A Series of Modern Surveys in Mathematics. - P. xii+436. - ISBN: 3-540-00832-2.

[5] Cheltsov I., Przyjalkowski V., Shramov C. Fano threefolds with infinite automorphism groups // Izv. Math. - 2019. - Vol. 83, no. 4. - P. 860-907.

[6] Fujiki A. On automorphism groups of compact Kähler manifolds // Invent. Math. - 1978. - Vol. 44, no. 3. - P. 225-258.

[7] Huybrechts D. Lectures on K3 surfaces, - Cambridge University Press, Cambridge, 2016. - Vol. 158 of Cambridge Studies in Advanced Mathematics. - P. xi+485. — ISBN: 978-1-107-15304-2, — Access mode: https://doi.org/10.1017/CB09781316594193.

[8] Kim J. H. Jordan property and automorphism groups of normal compact Kähler varieties // Commun. Contemp. Math. — 2018. - Vol. 20, no. 3. P. 1750024, 9.

[9] Kodaira K. On compact analytic surfaces. II // Ann. of Math. (2). - 1963. Vol. 77. - P. 563-626.

[10] Kollár J. The structure of algebraic threefolds: an introduction to Mori's program // Bull. Amer. Math. Soc. (N.S.). 1987. - Vol. 17, no. 2. - P. 211-273. - Access mode: https://doi.org/10.1090/S0273-0979-1987-15548-0.

[11] Maruyama M. On automorphism groups of ruled surfaces // J. Math. Kyoto Univ. - 1971. - Vol. 11. - 89-112.

[12] Miranda R. The basic theory of elliptic surfaces. Dottorato di Ricerca in Matematica. [Doctorate in Mathematical Research]. — ETS Editrice, Pisa, 1989. - P. vi+108.

[13] Mukai Sh., Namikawa Yu. Automorphisms of Enriques surfaces which act trivially on the cohomology groups // Invent. Math. - 1984. - Vol. 77, no. 3. P. 383-397. - Access mode: https://doi.org/10.1007/BF01388829.

[14] Nikulin V. Classification of Picard lattices of $K 3$-surfaces // Izv. Math. 2018. - Vol. 82, no. 4. - P. 752-816.

[15] Prokhorov Yu., Shramov C. Jordan property for groups of birational selfmaps // Compositio Math. — 2014. — Vol. 150, no. 12. — P. 2054-2072.

[16] Prokhorov Yu., Shramov C. Automorphism groups of compact complex surfaces // https://doi.org/10.1093/imrn/rnz124 — 2020.

[17] Prokhorov Yu., Shramov C. Finite groups of birational selfmaps of threefolds // Math. Res. Lett. — 2018. — Vol. 25, no. 3. - P. 957-972.

[18] Pukhlikov A. Automorphisms of certain affine complements in the projective space // Sb. Math. - 2018. - Vol. 209, no. 2. - P. 276-289.

[19] Sawon J. Isotrivial elliptic K3 surfaces and Lagrangian fibrations // arXiv:1406.1233. - 2014.

[20] Serre J.-P. Bounds for the orders of the finite subgroups of $G(k) / /$ Group representation theory. - EPFL Press, Lausanne, 2007. - P. 405-450.

[21] Shramov C. Finite groups acting on elliptic surfaces // https://doi.org/10.1007/s40879-019-00383-y — 2019.

[22] Shramov C., Vologodsky V. Automorphisms of pointless surfaces // arXiv:1807.06477. - 2018.

[23] Ueno K. Classification theory of algebraic varieties and compact complex spaces. Lecture Notes in Mathematics, Vol. 439. - Springer-Verlag, BerlinNew York, 1975. - P. xix +278. - Notes written in collaboration with P. Cherenack. 
Yuri Prokhorov

Steklov Mathematical Institute of RAS, 8 Gubkina street, Moscow 119991, Russia. prokhoro@mi-ras.ru

\section{Constantin Shramov}

Steklov Mathematical Institute of RAS, 8 Gubkina street, Moscow 119991, Russia. costya.shramov@gmail.com 